\title{
Level V Clearance in Neck Dissection for Papillary Thyroid Carcinoma: A Need for Homogeneous Studies
}

\author{
Azhar Jan Battoo ${ }^{1}$ Zahoor Ahmad Sheikh ${ }^{2}$ Krishnakumar Thankappan ${ }^{3}$ Abdul Wahid Mir ${ }^{2}$ \\ Altaf Gowhar Haji ${ }^{4}$
}

${ }^{1}$ Department of Surgical Oncology (Head and Neck Services), Sher i Kashmir Institute of Medical Sciences, Srinagar,

Jammu and Kashmir, India

2 Department of Surgical Oncology, Sher i Kashmir Institute of Medical

Sciences, Srinagar, Jammu and Kashmir, India

${ }^{3}$ Department of Head and Neck Surgical Oncology, Amrita Institute of Medical Sciences, Cochin, Kerala, India

${ }^{4}$ Department of Head Surgical Oncology, Sher i Kashmir Institute of Medical Sciences, Srinagar, Jammu and Kashmir, India

\begin{abstract}
Address for correspondence Azhar Jan Battoo, MCh, Department of Surgical Oncology (Head and Neck Services), Sher i Kashmir Institute of Medical Sciences, Srinagar-190011, Jammu and Kashmir, India (e-mail: azatmsy@gmail.com).
\end{abstract}

Int Arch Otorhinolaryngol 2018;22:449-454.

\begin{abstract}
Introduction Papillary thyroid carcinoma has a very high rate of lateral neck node metastases, and there is almost unanimity concerning the fact that some sort of formal neck dissection must be performed to address the clinical neck disease in these cases. Although there is an agreement that levels II to IV need to be cleared in these patients, the clearance of level $\mathrm{V}$ is debatable.

Objectives We herein have tried to analyze various papers that have documented a structured approach to neck dissection in these patients. Moreover, we have also tried to consider this issue through various aspects, like spinal accessory nerve injury and the impact of neck recurrence on survival.

Data Synthesis The PubMed, Medline, Google Scholar, Surveillance, Epidemiology, and End Results (SEER), and Ovid databases were searched for studies written in English

\section{Keywords}

- papillary thyroid carcinoma

- spinal accessory nerve

- neck dissection that focused on lateral neck dissection (levels II-IV or II-V) for papillary thyroid carcinoma. Case reports with 10 patients or less were excluded.

Conclusions The current evidence is equivocal whether to clear level $\mathrm{V}$ or not, and the studies published on this issue are very heterogeneous. Level IIIIV versus level II-V selective neck dissections in node-positive papillary thyroid carcinoma patients is far from categorical, with pros and cons for both approaches. Hence, we feel that there is a need for more robust homogeneous data in order to provide an answer to this question.
\end{abstract}

\section{Introduction}

Papillary thyroid carcinoma (PTC) is the most common thyroid malignancy, and it has an excellent prognosis with, 10 -year overall survival rates of $95 \%$ to $98 \% .{ }^{1}$ However, PTC has a propensity for lymphatic metastases, and $23 \%$ to $56 \%$ of the patients present with palpable neck nodes. ${ }^{2}$ The impact of central and lateral neck metastases on prognosis is not the same. This is reflected in the American Joint Committee of Cancer (AJCC) staging to differentiate thyroid cancer patients older than 45 years of age, in which patients with lateral neck disease are classified as stage IV, and patients with central received

January 30, 2017

accepted

October 23, 2017

published online

December 13, 2017
DOI https://doi.org/

10.1055/s-0037-1608909.

ISSN 1809-9777.
Copyright $\odot 2018$ by Thieme Revinter

Publicações Ltda, Rio de Janeiro, Brazil
License terms

() (i) $\ominus$ (\$) 
neck disease are classified as stage III. There is a controversy regarding prophylactic central neck node dissection in patients with PTC, but there is near consensus against performing prophylactic lateral neck dissection in PTC patients.

The pattern of lymph node involvement in PTC has been evaluated in various studies, and levels II, III, IV, and VI have been found to be the most frequently involved nodal basins. Hence, the experts in the field of thyroid surgery agree that these levels need to be addressed in any sort of neck dissection performed to clear the clinical neck disease. Similarly, the published literature does not support addressing level I in PTC patients, as this level is rarely involved in PTC patients with lateral neck disease. The debate about the clearance of level V in these patients has been simmering for a long time, and in the present review we have tried to answer this question. We will try to address this question under following headings:

a) Does lateral neck node involvement have any bearing on locoregional recurrence, disease-free survival (DFS) and overall survival (OS)?

b) How effectively can we detect affected neck nodes preoperatively?

c) Does the extent of neck dissection have any bearing on regional recurrence and survival?

d) What are the incidence and severity of shoulder dysfunction associated with accessory spinal nerve dissection in different types of neck dissections?

e) What is the incidence of the nodal involvement of level V in the published data, and how has it been addressed?

\section{Review of Literature}

The PubMed, Medline, Google Scholar, Surveillance, Epidemiology, and End Results (SEER), and Ovid databases were searched for the terms papillary thyroid carcinoma, selective neck dissection, level $V$, spinal accessory nerve, and neck recurrence, and relevant papers were identified. Only papers written in English were included in the present review. Studies that focused on lateral neck dissection (levels II-IV or II-V) for PTC were included. Case reports with 10 patients or less were excluded. Articles that focused on prophylactic neck dissection, or medullary or anaplastic carcinoma, were also excluded.

\section{Discussion}

\section{Lateral Neck Node Involvement and Recurrence}

The influence of nodal metastasis on local recurrence, DFS and OS has been variably reported. A multivariate analysis of 9,904 patients with differentiated thyroid carcinoma (DTC) with neck nodes, registered in the SEER database, demonstrated that age $>45$ years, presence of distant metastasis, large tumor size, and lymph node involvement significantly predicted a poor outcome. The OS rates at 14 years were $82 \%$ for nodenegative and $79 \%$ for node-positive patients $(p<0.05){ }^{3}$ A follow-up analysis of the SEER database identified 33,088 patients with DTC, and the analysis showed that lymph node disease in patients with papillary carcinoma older than 45 years of age did not influence survival $(p=0.535) .{ }^{4}$ In patients with PTC with pathologically-proven cervical lymph node metastases, the median risk of loco-regional lymph node recurrence varies with nodal status, with recurrence rates for patients who are initially clinically N0 of $2 \%$ (range: $0-9 \%$ ) against $22 \%$ (range: $10-42 \%)$ for those with nodal involvement at presentation. Furthermore, the median risk of recurrence in these patients varies markedly with the number of involved nodes: $4 \%$ for $<5$ nodes against $19 \%$ for $>5$ nodes. ${ }^{5}$ The categorization of node-positive patients with DTC aged $<45$ years as stage I according to the 7th edition of the AJCC's Cancer Staging Manual is probably a testimony to the fact that nodal disease in younger age groups does not have any bearing on survival.

Imaging Exams in Cases of Lateral Neck Lymph Nodes Neck lymph nodes have been evaluated with various imaging modalities; however, ultrasonography (USG) is the most common imaging technique used to evaluate neck nodes, and it is a very accurate tool to detect neck nodes suspicious for harboring disease. The various USG features that help predict malignancies in neck nodes include: micro-calcifications (sensitivity: 5-69\%; specificity: 93-100\%); the cystic nature of the node (sensitivity: 10-34\%; specificity: 91-100\%); peripheral vascularity (sensitivity: 40-86\%; specificity: 57-93\%); hyperechogenicity (sensitivity: $30-87 \%$; specificity: $43-95 \%$ ); and a round shape (sensitivity: $37 \%$; specificity: $70 \%{ }^{6}{ }^{6}$ Hence, with such a high degree of precision in expert hands, the imaging modalities in vogue in the current head and neck surgical practice allow us to detect specific levels of nodes involved and tailor our neck dissection accordingly. Moreover, fine needle aspiration cytology (FNAC) with or without measurement of thyroglobulin in needle washouts from the suspicious node can further clear the doubt regarding the presence of malignancy. ${ }^{7}$ From the aforementioned information, it is clear that therapeutic neck dissection can be precisely planned in PTC patients to address the levels involved.

\section{Type of Neck Dissection and Recurrence}

Turanli $^{8}$ assessed the impact of the extent of the neck dissection on regional recurrence in PTC patients. Almost all patients evaluated had PTC (61 patients in total, and 2 patients had follicular thyroid carcinoma). The patients were evaluated either by FNAC, frozen sections or excisional biopsy. All patients received radioactive iodine (RAI). The patients were analyzed by dividing them into two groups: the selective neck dissection group (group I), in which levels II-IV were dissected $(n=29)$, and the comprehensive neck dissection group (group II), which included patients who had undergone radical neck dissection (RND) or modified radical neck dissection (MRND) $(n=32)$. Neck dissections were performed in 37 patients $(60.7 \%)$ during their first surgery, in 21 patients (34.4\%) during their second surgery (for nodal recurrence), and in 3 patients (4.9\%) for nodal recurrence after initial neck exploration. A total of 9 patients had distant metastases at presentation. Lymph node recurrence developed in 9 patients in group I and in 7 patients in group II. Thus, the type of neck dissection performed did not show any differential effect on local recurrence, DFS and OS.

Mazzaferri and Young, ${ }^{9}$ in their analysis of 576 PTC patients, found no statistically significant difference in recurrence or death rates between patients who underwent non-anatomical 
dissection and those who underwent comprehensive neck dissection (RND or MRND) for nodal metastases. Bhattacharyya ${ }^{10}$ reviewed 2,097 PTC patients, and failed to demonstrate any significant advantage of RND or MNRD over selective neck dissection (SND) vis-à-vis local recurrence time, local recurrence rate, DFS and OS.

From the aforementioned information we can conclude that comprehensive therapeutic neck dissection involving level V dissection does not lead to less regional recurrences, nor does it have any survival advantages in PTC patients.

\section{Shoulder Dysfunction with Selective and Comprehensive Neck Dissections}

Dissection of the spinal accessory nerve during neck dissection is associated with shoulder dysfunction, and MRND entails more nerve dissection than SND. Chepeha et al ${ }^{11}$ evaluated the shoulder function morbidity after neck dissection by comparing the functional assessment of the shoulder in two groups of patients; one undergoing MRND and another undergoing SND. Constant Shoulder Test (CST), which is a weighted test that combines patient symptom scores (35\%) and objective measures of active shoulder function (65\%), was used for the functional assessment of the shoulder function. Patients with MRND had significantly worse shoulder function than SND patients. Cappiello et $\mathrm{al}^{12}$ evaluated shoulder disability after different types of selective neck dissections (levels II-IV versus levels II-V) and concluded that clearance of the posterior triangle of the neck increases shoulder morbidity. Hence, dissecting the accessory nerve in the posterior triangle is associated with increased morbidity.

\section{Pattern of Metastases to Neck Lymph Nodes and Level $\mathrm{V}$ Involvement}

Sivanandan and Soo ${ }^{13}$ evaluated the pattern of lymph node metastases in 70 PTC patients who underwent 80 neck dissections. Therapeutic neck dissection was performed if the neck nodes were palpable upon physical examination, regardless of any additional imaging for further evaluation. Comprehensive neck dissection involving dissection of levels I-V with or without sacrifice of the sternocleidomastoid muscle, the internal jugular vein and/or the spinal accessory nerve was performed. Level I lymph nodes were only present in 3 out of 80 specimens. Level $V$ was positive in 23 out of 80 neck dissection specimens; however, level V nodes were not segregated into sublevels Va and $\mathrm{Vb}$. Moreover, the authors did not comment on the percentage of level $\mathrm{V}$ positive nodes in clinically negative level $\mathrm{V}$ necks. Level $\mathrm{V}$ was not involved in isolation, although 14 (18\%) specimens had single level diseases.

Pingpank et al ${ }^{14}$ evaluated 44 PTC patients who underwent 51 comprehensive neck dissections involving levels I through V. Although it was not explicitly mentioned, one can discern from the text that a clinically palpable disease was set as a criterion for neck dissection. A total of 8 specimens (16\%) were negative for pathology on the final histopathological analysis. Only 16 specimens were labeled separately for level I. Overall, $12 \%$ of level I nodes were positive for malignancy. Out of 51 neck dissections, 34 neck dissections had level IIb labeled separately, which was positive in 7 (21\%) out of 34 specimens, and this was the sole disease present at level II in 3 specimens. Disease at only 1 level was found in 14 neck specimens (32\%). Level $\mathrm{V}$ was positive for metastases in 14 out of 50 specimens. Lateral neck control was achieved in $35(80 \%)$ out of 44 necks. Like the previous study by Sivanandan and Soo, the study sample was composed of new and previously treated cases.

Kupferman et $\mathrm{al}^{15}$ studied 39 PTC patients, in whom 44 neck dissections involving levels II through $\mathrm{V}$ were performed. The study involved an evaluation of both new and recurrent cases. All patients were evaluated before surgery by FNAC or radiologic examination (the specific imaging modality used was not mentioned), and the disease was confirmed either by FNAC or by a previous biopsy. Out of 44 neck dissection specimens, 4 were negative for malignancy. Levels I and V were involved in 1/7 (14\%) and 9/44 (20\%) neck dissection specimens. Level I was dissected only if the radiographic, pathologic, or intraoperative findings were suggestive of cancer. A total of 14 patients only had the disease at 1 level, 5 patients only had the disease at level II, and 3 patients each had the disease at levels III, IV, or V. Since level V was not divided into sublevels, no distinction could be made as to how many had involvement of levels $\mathrm{Va}, \mathrm{Vb}$ or both.

Lee et al $^{16}$ evaluated 46 newly diagnosed cases of PTC, with the clinical disease documented by USG, computed tomography (CT) and FNAC. A total of 55 neck dissections involving levels II through $\mathrm{V}$ were performed. Although level V was involved in 11 (20\%) specimens, it was not involved alone in any of the specimens. The incidence of lymph node metastasis at level IIb was of $22 \%$ ( 12 specimens). Out of 12 specimens with metastatic lymph node at level IIb, 11 (92\%) also had the disease at level Ila. The rate of level IIb lymph node involvement in patients with metastatic lymph nodes at level IIa was of $34 \%$ (11 out of 32 ), but only 1 specimen was positive for the disease in absence of disease at level Ila. Once again, this study failed to make any distinction between the various sublevels of level $\mathrm{V}$, and it showed high involvement of level IIb.

Ahn et $\mathrm{al}^{17}{ }^{17}$ in their report of 37 patients undergoing total thyroidectomy and lateral $(n=28)$ or central $(n=9)$ neck dissections, noted the high accuracy of the CT and USG, with sensitivity of $100 \%$ and $100 \%$, specificity of $90 \%$ and $80 \%$, positive predictive value of $96 \%$ and $93 \%$, negative predictive value of $100 \%$ and $100 \%$, and accuracy of $97 \%$ and $95 \%$ respectively. Level V was only positive in 4 out of 26 (15\%) neck specimens. For Level V, the positive predictive value of both the USG and the CT was of $100 \%$. One wonders that, with such a high diagnostic accuracy, why it is not possible to dissect levels that do not have such high percentages of involvement, like IIb, V, only if they are highly suspicious upon imaging studies.

Khafif et $\mathrm{al}^{18}$ studied 37 PTC patients, 11 of which had previously had their thyroids operated without addressing the neck. Metastasis was confirmed by USG and FNAC of the nodes. Levels II-IV were dissected in 31 patients, and levels II$\mathrm{V}$, in 6 patients. Level $\mathrm{V}$ was dissected only when it was involved or when retrojugular nodes were involved; however, the study does not mention the diagnostic accuracy of the USG at this level. Level V was only involved in 5 patients. 
Kupferman et $\mathrm{al}^{19}$ evaluated the predictors of level $\mathrm{V}$ metastasis in 70 DTC patients who underwent 75 neck dissections that ranged from RND to SND. Previously treated as well as new cases were included, and 33\% had prior neck dissections, whereas $31 \%$ had prior neck biopsies. There were six cases of follicular carcinoma. The sensitivity, specificity, positive predictive value and negative predictive value for the USG and $\mathrm{CT} / \mathrm{MRI}$ were $21 \%, 70 \%, 33 \%, 56 \%$ and $15 \%, 90 \%, 80 \%, 30 \%$ respectively. A total of $53 \%$ of ipsilateral neck specimens had positive level $\mathrm{V}$, and $57 \%$ of contralateral neck specimens had the disease. The presence of ipsilateral level $\mathrm{V}$ metastases was significantly associated with multifocal disease $(p<0.05)$, and with ipsilateral levels II $(p<0.05)$, III $(p<0.05)$, or IV $(p<0.01)$ metastases. The ipsilateral involvement of level $\mathrm{V}$ was also associated with contralateral lymph node metastases $(p<0.05)$. Isolated level $\mathrm{V}$ positivity was only observed in 5\% of the specimens. One of the biggest drawbacks of this study vis-à-vis the pattern of nodal metastasis in PTC is that the study included patients with operated necks, as well as six follicular thyroid carcinoma patients.

Lee et $\mathrm{al}^{20}$ analyzed 167 PTC patients who underwent 191 MRNDs. The imaging technique used to evaluate the neck nodes was not documented. The MRNDs addressed levels II through $\mathrm{V}$, and were performed upfront in $73 \%$ of the necks; the remaining $23 \%$ were recurrent necks. Level IIb and $V$ nodes were involved in $6.8 \%$ and $16.8 \%$ of neck dissection specimens respectively. Level IIb metastasis was always found in association with level Ila nodes, and it was observed in cases showing aggressive nodal metastasis. No isolated level $\mathrm{V}$ metastasis was documented. This study did not detail the handling of level $\mathrm{V}$ nodes, and the sublevels of level V were not discussed.

Roh et al $^{21}$ evaluated 52 PTC patients who underwent 57 MRNDs (from levels Ib to V). All the patients were new cases, and they all underwent USG and FNAC before surgery. Level Va was divided into sublevels Vas (superior to the spinal accessory nerve) and Vai (between the spinal accessory nerve and the transverse line drawn at the level of the cricoid). Out of 54 ipsilateral necks, 9 and 7 necks were positive for level IIb and Vai (13\%). Levels V and IIb were not involved in isolation, and were always associated with disease at other levels. Levels Vai and $\mathrm{Vb}$ were positive for metastasis in 7 (13\%) and 2 (3.7\%) specimens respectively; however both the $\mathrm{Vb}$-positive cases had negative Vai nodes. This is one of the few studies that mentioned in detail various sublevels of level $\mathrm{V}$ and showed that level $\mathrm{V}$ is not so commonly involved.

Yanir and Doweck ${ }^{22}$ evaluated 27 well-differentiated thyroid carcinoma patients who underwent 28 selective neck dissections. The patients underwent a physical examination and a CT scan, and 16 had upfront neck dissections, whereas 11 had neck dissection after primary thyroid surgery. Three patients had follicular carcinoma. All of the the patients received RAI; hence, interval neck dissection patients (those operated for neck pathology after a varying time interval after primary thyroidectomy) received it prior to the neck dissection. A total of 24 patients ( 25 necks) underwent SND including levels II through VI, and 3 patients had SND including levels IIIV and VI. There were 2 level IIb metastasis, 1 of them without the involvement of level Ila. Level V was involved in $20 \%$ of the neck dissections; 4 out of 6 necks that were positive for level $V$ metastasis also had level IV metastasis, and 2 patients were positive at level $\mathrm{V}$ without level IV involvement. This study had a heterogeneous patient population and limited patient number.

Farrag et $\mathrm{al}^{23}$ analyzed 53 PTC patients who underwent therapeutic lateral neck dissection that included levels IIa and IIb, and/or Va and Vb. Only 10 of these patients were new cases: all of the others were either recurrent cases or cases with residual disease. Metastasis was confirmed by USGguided FNAC after an initial evaluation with USG for suspicious nodes. A total of 60 neck dissections were performed, and level II was positive in 33/59 neck dissections, level IIb was only positive in 5 specimens and that too with level IIa involvement. Level $\mathrm{V}$ was excised 40 times and was positive in 16 specimens $(16 / 40=40 \%)$. Level Va was not positive in any of the specimens. This study did not mention the criteria used to decide whether or not to treat level $\mathrm{V}$ in the patients.

Koo et $\mathrm{al}^{24}$ evaluated 76 PTC patients using USG/CT and FNAC. There were 57 new patients and 19 recurrent patients, all of whom underwent therapeutic lateral neck dissection of levels II through $\mathrm{V}$. Level $\mathrm{V}$ metastasis was present in 12 out of $76(15.8 \%)$ neck dissection specimens. Out of 40 positive level II necks, 31 were positive for level IIb, 1 was positive for IIa, and 8 were positive for IIa and IIb. Like many previous studies, this one addressed both new and recurrent cases, and did not have a high percentage of level $\mathrm{V}$ involvement. Additionally, level $\mathrm{V}$ was not reported in terms of sublevel involvement.

Lee et $\mathrm{al}^{25}$ studied 70 PTC patients ( 80 necks), and 11 of those patients developed delayed neck metastasis and were operated secondary to recurrence. The USG criteria were established as follows: the patients were divided into 3 groups: lymph node minor diameter $\geq 5 \mathrm{~mm}, \geq 6 \mathrm{~mm}$, and $\geq 7 \mathrm{~mm}$, with a fixed minor/major axis ratio of 0.5 or more. Furthermore, the minor/major axis ratio was established as 0.4 or more, 0.5 or more, and 0.6 or more, with a fixed minor axis of $6 \mathrm{~mm}$ or more. In each group, the sensitivity, accuracy, and specificity of the parameter established were calculated. A minor axis of $6 \mathrm{~mm}$ or more and a minor/major axis ratio of 0.5 or more were established as appropriate diagnostic criteria. The detection rate of the USG at level V was of $29 \%$ (4/14), when compared with histopathologic diagnosis. Even though this study aimed at establishing diagnostic USG criteria, it provides us with a very important statistic about the detection rate of USG for metastasis at level V. However, we should note that there is no consensus regarding the criteria established for the detection of metastases. With the USG criteria in vogue today, ${ }^{26}$ we should expect a much higher detection rate, and that can help physicians tailor neck dissections accordingly.

Lim et $\mathrm{al}^{27}$ evaluated 70 PTC patients for occult disease at level V. A total of 56 patients underwent primary thyroidectomy and levels II-V neck dissection, while 14 patients had salvage neck dissection for recurrence. None of the patients had any suspicious nodes detected by USG at level V. Occult metastases at level V were observed in 11 (16\%) patients. According to the level V subgroups, 8 patients 
had occult lymph node metastases at level Va, 4 patients had occult metastases at level $\mathrm{Vb}$, and 1 patient had positive lymph nodes at levels $\mathrm{Va}$ and $\mathrm{Vb}$. Level $\mathrm{V}$ was always involved in association with level IV, and there were no isolated level V metastases. No positive level $\mathrm{V}$ lymph nodes were found in 19 patients without suspicious metastatic lymph nodes at level IV. All of the 11 patients (22\%) confirmed as having a metastatic level $\mathrm{V}$ lymph node were histopathologically among the 51 patients who had suspicious level IV lymph node metastasis on the preoperative USG. This study probably hints at doing a more dedicated USG of the neck, particularly at levels IV and V, to further modify the levels to be included in neck dissection.

Vayisoglu et $a^{28}$ performed 47 neck dissections on 33 consecutive PTC patients. Levels II to V neck dissections were performed, and level I was added if, during surgery, the nodes at that level were felt to be significant. Out of 33 patients, 3 were recurrent. The patients were evaluated by palpation and/or USG and CT. Only 22 (46.8\%) out of 47 neck dissections were positive for metastases, and $18 \%$ of level $\mathrm{V}$ dissections were positive for metastases in node positive necks. Level IIb nodes were positive in only 1 out of 22 (4.5\%) positive neck dissections. Considering all neck dissections, levels V and IIb were only positive in $8.5 \%$ and $2.1 \%$ of the specimens respectively. This study had an unusually low histological positive yield for necks operated upon with therapeutic purposes, and showed very low involvement of level V.

Yuce et $\mathrm{al}^{29}$ studied the regional metastatic pattern in 48 PTC patients who underwent 61 neck dissections (levels IIVI). Seven patients had previously performed subtotal thyroidectomy, and underwent completion thyroidectomy and neck dissection. The patients underwent USG, CT, and USGguided FNAC per operative frozen section. Level II was positive in 28/61(46\%) specimens, while level $\mathrm{V}$ was positive in $21 / 61$ (34\%) neck specimens. This study did not discuss the various level $\mathrm{V}$ sublevels, even though it showed a high percentage of involvement of level $\mathrm{V}$.

King et $\mathrm{al}^{30}$ studied the management of lateral cervical metastases in 32 PTC patients who underwent 39 level II to VI neck dissections. A total of 14 patients were operated for recurrent neck pathology. The patients underwent pre-operative USG and CT/MRI, and metastases were confirmed by FNAC. Level I nodes were dissected in six specimens based on preoperative imaging evidence or perioperative findings. Level Ila was involved in 49\% (19/39); level IIb, in 62\% (24/39); level $\mathrm{Va}$, in $8 \%$ (3/39); and level Vb, in 31\% (12/39) of neck specimens. In $3 / 6$ patients (50\%) who underwent a level I lymph node dissection, the specimen contained metastatic disease. Like the majority of the aforementioned studies, this one showed a a low involvement of level Va, but, unlike other studies, it showed very high rates of level II metastases (62\%).

Merdad et al $^{31}$ published the largest series on the pattern of neck nodes in PTC patients. They evaluated 185 patients with lateral neck node involvement confirmed by FNAC who underwent 248 neck dissections. A total of $44 \%$ of the patients underwent selective neck dissection of levels II through $\mathrm{Vb}$ for recurrent disease in the lateral neck, and 56\% underwent neck dissection at the time of their total thyr- oidectomy. Levels II, III, IV, and Vb were found to harbor metastases in 122 (49\%), 189 (76\%), 152 (61\%), and 72 (29\%) patients respectively. Merdad et al, while describing their approach to the neck, detailed that they approach the posterior triangle of the neck at the level of the spinal accessory nerve and dissect caudally. According to the American Head and Neck Society consensus statement on the classification and terminology of neck dissection, ${ }^{32}$ the area between the spinal accessory nerve and the transverse line drawn at the level of the cricoid is part of level Va; therefore, we think the authors probably included a much larger field of dissection at level $\mathrm{Vb}$ and included a part of sublevel Va. This study also failed to mention the number of patients who underwent RAI ablation prior to developing a secondary disease in the neck.

Eskander et $\mathrm{al}^{33}$ performed a meta-analysis that detailed the pattern of lateral lymph node involvement in nodepositive necks of PTC patients. Before we comment on this meta-analysis, it is worthwhile to mention the $\mathrm{I}^{2}$ statistic, which is a static quantity described by Higgins et al, ${ }^{34}$ ranging from $0-100 \%$, that measures the degree of inconsistency across studies in a meta-analysis. The $\mathrm{I}^{2}$ statistic describes the percentage of variation across studies that is due to heterogeneity rather than chance. In the meta-analysis by Eskander et al, the degree of heterogeneity between various studies is high, and for level $\mathrm{V}$, the $\mathrm{I}^{2}$ is of $75.97 \%$. Even though the authors performed a random model meta-regression analysis of various factors, and observed that none of these factors explained or decreased the observed heterogeneity estimates leading to insignificant clinical implications, in our opinion, the degree of heterogeneity is too high, and has to be considered as one of the limitations of the study. The bigger question, the one about the recurrence at level $\mathrm{V}$ when this level is not suspected to be involved preoperatively and subsequently not addressed, has not been documented in well-conducted studies. When such a thing is not well-documented, quality of life issues have a bigger role to play. Cappiello et $\mathrm{al}^{12}$ evaluated shoulder disability after different selective neck dissections (levels II-IV versus levels II-V), and concluded that clearance of the posterior triangle of the neck increases shoulder morbidity.

\section{Final Comments}

To conclude, the final answer to level II-IV versus level II-V selective neck dissection in node-positive PTC patients is far from categorical, with pros and cons for both approaches. In our opinion, an analysis of large multi-institutional data with more homogeneity, as well as detailing the recurrence pattern in the ipsilateral neck may provide a more robust answer.

Conflicts of Interest

The authors have no conflicts of interest to disclose.

Financial Support

This research received no specific grant from any funding agency whatsoever. 


\section{Authors' Contributions}

AJB designed the review, performed the literature review, drafted the manuscript, and made the evidence-based summaries. SZA and AWM revised the manuscript, reviewed the summaries, and contributed to the literature search. KT and AGH designed the review and revised the manuscript and reviewed the summaries. All authors read and approved the final manuscript.

\section{References}

1 Nikiforov YE, Nikiforova MN. Molecular genetics and diagnosis of thyroid cancer. Nat Rev Endocrinol 2011;7(10):569-580

2 Wang TS, Dubner S, Sznyter LA, Heller KS. Incidence of metastatic well-differentiated thyroid cancer in cervical lymph nodes. Arch Otolaryngol Head Neck Surg 2004;130(01):110-113

3 Podnos YD, Smith D, Wagman LD, Ellenhorn JD. The implication of lymph node metastasis on survival in patients with well-differentiated thyroid cancer. Am Surg 2005;71(09):731-734

4 Zaydfudim V, Feurer ID, Griffin MR, Phay JE. The impact of lymph node involvement on survival in patients with papillary and follicular thyroid carcinoma. Surgery 2008;144(06):1070-1077, discussion 1077-1078

5 Randolph GW, Duh QY, Heller KS, et al; American Thyroid Association Surgical Affairs Committee's Taskforce on Thyroid Cancer Nodal Surgery. The prognostic significance of nodal metastases from papillary thyroid carcinoma can be stratified based on the size and number of metastatic lymph nodes, as well as the presence of extranodal extension. Thyroid 2012;22(11):1144-1152

6 Cooper DS, Doherty GM, Haugen BR, et al; American Thyroid Association (ATA) Guidelines Taskforce on Thyroid Nodules and Differentiated Thyroid Cancer. Revised American Thyroid Association management guidelines for patients with thyroid nodules and differentiated thyroid cancer. Thyroid 2009;19(11):1167-1214

7 Snozek CL, Chambers EP, Reading CC, et al. Serum thyroglobulin, high-resolution ultrasound, and lymph node thyroglobulin in diagnosis of differentiated thyroid carcinoma nodal metastases. J Clin Endocrinol Metab 2007;92(11):4278-4281

8 Turanli S. Is the type of dissection in lateral neck metastasis for differentiated thyroid carcinoma important? Otolaryngol Head Neck Surg 2007;136(06):957-960

9 Mazzaferri EL, Young RL. Papillary thyroid carcinoma: a 10 year follow-up report of the impact of therapy in 576 patients. Am J Med 1981;70(03):511-518

10 Bhattacharyya N. Surgical treatment of cervical nodal metastases in patients with papillary thyroid carcinoma. Arch Otolaryngol Head Neck Surg 2003;129(10):1101-1104

11 Chepeha DB, Taylor RJ, Chepeha JC, et al. Functional assessment using Constant's Shoulder Scale after modified radical and selective neck dissection. Head Neck 2002;24(05):432-436

12 Cappiello J, Piazza C, Giudice M, De Maria G, Nicolai P. Shoulder disability after different selective neck dissections (levels II-IV versus levels II-V): a comparative study. Laryngoscope 2005;115(02):259-263

13 Sivanandan R, Soo KC. Pattern of cervical lymph node metastases from papillary carcinoma of the thyroid. Br J Surg 2001;88(09):1241-1244

14 Pingpank JF Jr, Sasson AR, Hanlon AL, Friedman CD, Ridge JA. Tumor above the spinal accessory nerve in papillary thyroid cancer that involves lateral neck nodes: a common occurrence. Arch Otolaryngol Head Neck Surg 2002;128(11):1275-1278

15 Kupferman ME, Patterson M, Mandel SJ, LiVolsi V, Weber RS. Patterns of lateral neck metastasis in papillary thyroid carcinoma. Arch Otolaryngol Head Neck Surg 2004;130(07):857-860
16 Lee BJ, Wang SG, Lee JC, Son SM, Kim IJ, Kim YK. Level IIb lymph node metastasis in neck dissection for papillary thyroid carcinoma. Arch Otolaryngol Head Neck Surg 2007;133(10):1028-1030

17 Ahn JE, Lee JH, Yi JS, et al. Diagnostic accuracy of CT and ultrasonography for evaluating metastatic cervical lymph nodes in patients with thyroid cancer. World J Surg 2008;32(07):1552-1558

18 Khafif A, Ben-Yosef R, Abergel A, Kesler A, Landsberg R, Fliss DM. Elective paratracheal neck dissection for lateral metastases from papillary carcinoma of the thyroid: is it indicated? Head Neck 2008;30(03):306-310

19 Kupferman ME, Weinstock YE, Santillan AA, et al. Predictors of level $\mathrm{V}$ metastasis in well-differentiated thyroid cancer. Head Neck 2008;30(11):1469-1474

20 Lee J, Sung TY, Nam KH, Chung WY, Soh EY, Park CS. Is level IIb lymph node dissection always necessary in N1b papillary thyroid carcinoma patients? World J Surg 2008;32(05):716-721

21 Roh JL, Kim JM, Park CI. Lateral cervical lymph node metastases from papillary thyroid carcinoma: pattern of nodal metastases and optimal strategy for neck dissection. Ann Surg Oncol 2008;15 (04):1177-1182

22 Yanir Y, Doweck I. Regional metastases in well-differentiated thyroid carcinoma: pattern of spread. Laryngoscope 2008;118 (03):433-436

23 Farrag T, Lin F, Brownlee N, Kim M, Sheth S, Tufano RP. Is routine dissection of level II-B and V-A necessary in patients with papillary thyroid cancer undergoing lateral neck dissection for FNA-confirmed metastases in other levels. World J Surg 2009;33 (08):1680-1683

24 Koo BS, Yoon YH, Kim JM, Choi EC, Lim YC. Predictive factors of level IIb lymph node metastasis in patients with papillary thyroid carcinoma. Ann Surg Oncol 2009;16(05):1344-1347

25 Lee K, Kawata R, Nishikawa S, Yoshimura K, Takenaka H. Diagnostic criteria of ultrasonographic examination for lateral node metastasis of papillary thyroid carcinoma. Acta Otolaryngol 2010;130(01):161-166

26 Leenhardt L, Erdogan MF, Hegedus L, et al. 2013 European thyroid association guidelines for cervical ultrasound scan and ultrasound-guided techniques in the postoperative management of patients with thyroid cancer. Eur Thyroid J 2013;2(03):147-159

27 Lim YC, Choi EC, Yoon YH, Koo BS. Occult lymph node metastases in neck level V in papillary thyroid carcinoma. Surgery 2010;147 (02):241-245

28 Vayisoglu Y, Ozcan C, Turkmenoglu O, et al. Level IIb lymph node metastasis in thyroid papillary carcinoma. Eur Arch Otorhinolaryngol 2010;267(07):1117-1121

29 Yüce I, Cağli S, Bayram A, Karasu F, Güney E. Regional metastatic pattern of papillary thyroid carcinoma. Eur Arch Otorhinolaryngol 2010;267(03):437-441

30 King JM, Corbitt C, Miller FR. Management of lateral cervical metastases in papillary thyroid cancer: patterns of lymph node distribution. Ear Nose Throat J 2011;90(08):386-389

31 Merdad M, Eskander A, Kroeker T, Freeman JL. Metastatic papillary thyroid cancer with lateral neck disease: pattern of spread by level. Head Neck 2013;35(10):1439-1442

32 Robbins KT, Shaha AR, Medina JE, et al; Committee for Neck Dissection Classification, American Head and Neck Society. Consensus statement on the classification and terminology of neck dissection. Arch Otolaryngol Head Neck Surg 2008;134(05):536-538

33 Eskander A, Merdad M, Freeman JL, Witterick IJ. Pattern of spread to the lateral neck in metastatic well-differentiated thyroid cancer: a systematic review and meta-analysis. Thyroid 2013; 23(05):583-592

34 Higgins JP, Thompson SG, Deeks JJ, Altman DG. Measuring inconsistency in meta-analyses. BMJ 2003;327(7414):557-560 